JOURNAL OF THE

AMERICAN MATHEMATICAL SOCIETY

Volume 22, Number 2, April 2009, Pages 353-362

S 0894-0347(08)00627-9

Article electronically published on October 21, 2008

\title{
ROTATION NUMBERS FOR QUASIPERIODICALLY FORCED CIRCLE MAPS-MODE-LOCKING VS. STRICT MONOTONICITY
}

\author{
KRISTIAN BJERKLÖV AND TOBIAS JÄGER
}

\section{INTRODUCTION}

For an orientation-preserving circle homeomorphism $g: \mathbb{T}^{1} \rightarrow \mathbb{T}^{1}$ it is wellknown that the rotation number behaves strictly monotonically whenever $g$ has no periodic points. On the other hand, the only situation where mode-locking occurs (that is, the rotation number is stable with respect to perturbations) is when there exists a closed interval which is mapped into its own interior by some iterate of $g$, as in the case of a stable periodic orbit. If the rotation number only stays constant on one side, this corresponds to the existence of parabolic periodic points. In this paper, we show that exactly the same picture holds for quasiperiodically forced (qpf) orientation-preserving circle homeomorphisms, where $p, q$-invariant strips (as introduced in [2] and [3]) serve as natural analogues of periodic orbits.

We consider continuous maps $f: \mathbb{T}^{2} \rightarrow \mathbb{T}^{2}$ of the form

$$
f(\theta, x)=\left(\theta+\omega, f_{\theta}(x)\right),
$$

where $\omega \in[0,1] \backslash \mathbb{Q}$. In addition, we require all fiber maps $f_{\theta}$ to be orientationpreserving circle homeomorphisms. The class of all such maps will be denoted by $\mathcal{F}$. Further, we will use the notation $f_{\theta}^{n}(x)=\pi_{2} \circ f^{n}(\theta, x)$. Given any continuous lift $F: \mathbb{T}^{1} \times \mathbb{R} \rightarrow \mathbb{T}^{1} \times \mathbb{R}$ of $f \in \mathcal{F}$, the limit

$$
\rho(F):=\lim _{n \rightarrow \infty}\left(F_{\theta}^{n}(x)-x\right) / n
$$

exists and is independent of $\theta$ and $x$ [1]. The fibered rotation number of $f$ is defined as $\rho(f):=\rho(F) \bmod 1$. In order to state our results, we need the following notions:

Definition 1.1. Suppose $f \in \mathcal{F}$ and $F$ is a lift of $f$. We let

$$
F_{\varepsilon}(\theta, x):=\left(\theta+\omega, F_{\theta}(x)+\varepsilon\right)
$$

and say the rotation number is strictly monotone in $f$ if the map $\varepsilon \mapsto \rho\left(F_{\varepsilon}\right)$ is strictly monotone in $\varepsilon=0$. If $\varepsilon \mapsto \rho\left(F_{\varepsilon}\right)$ is constant in a neighbourhood of $\varepsilon=0$, we say $f$ is mode-locked.

For the notion of a $p, q$-invariant strip used in the follwing statement, see Section 2

Received by the editors August 10, 2006.

2000 Mathematics Subject Classification. Primary 37E45, 37B55.

Key words and phrases. Rotation numbers, mode-locking, quasiperiodically forced systems.

(C)2008 American Mathematical Society Reverts to public domain 28 years from publication 
Theorem A. Suppose $f \in \mathcal{F}$ has no $p, q$-invariant strip. Then the rotation number is strictly monotone in $f$. In particular, this holds whenever $\omega, \rho(f)$ and 1 are rationally independent 1

Note that the second statement in Theorem A is the answer to the question asked by Herman in [1, Section 5.18, p. 497].

Theorem B. Suppose $f \in \mathcal{F}$ is mode-locked. Then there exists a closed annulus, bounded by continuous curves, which is mapped into its own interior by some iterate of $f$.

For a description of the critical cases with one-sided monotonicity, we refer to Section 4 .

Remark 1.2. The above results remain valid when the underlying irrational rotation on the circle in (1.1) is replaced by an irrational translation (Kronecker twist) on the $d$-dimensional torus. The proofs go through with only obvious and minor modifications needed. Some of the preliminary statements we use (for example Theorems 2.3 and 2.4) are only stated in the literature for one single forcing frequency as well, but here again the results remain valid with basically the same proofs.

There is one well-known example for which the statement of Theorem A has been known, namely in the case of the (generalised) Harper map

$$
s_{E}: \mathbb{T}^{1} \times \overline{\mathbb{R}} \rightarrow \mathbb{T}^{1} \times \overline{\mathbb{R}}, \quad(\theta, x) \mapsto\left(\theta+\omega, V(\theta)-E-\frac{1}{x}\right),
$$

where $V: \mathbb{T}^{1} \rightarrow \mathbb{R}$ is a continuous function and $E \in \mathbb{R}$. By letting $x_{n}=u_{n+1} / u_{n}$, there is a 1-1 correspondence between orbits of (1.3) and formal eigenfunctions of the discrete Schrödinger operator $\left(H_{\theta} u\right)_{n}=-\left(u_{n+1}+u_{n-1}\right)+V(\theta+n \omega) u_{n}$. Note that by identifying $\overline{\mathbb{R}}$ with $\mathbb{T}^{1},(1.3)$ defines an element of $\mathcal{F}$ and we can therefore speak of the fibred rotation number of $s_{E}$. Due to a fruitful interplay between spectral theory and dynamical systems methods, the dynamics of (1.3) is quite well-understood. In particular, it is known that the function $E \mapsto \rho\left(S_{E}\right)$ is monotone and continuous, and maps $\mathbb{R}$ onto $[0,1]$. In fact, it can be shown that $\rho\left(s_{E}\right)=k(E)$, where $k(E)$ denotes the integrated density of states for the corresponding Schrödinger operator. Moreover, if $\rho\left(s_{E}\right)$ is constant on an interval, then $\rho\left(s_{E}\right)=k \omega \bmod 1$ for some integer $k$ [4, 5]. In particular, if $\rho\left(s_{E}\right)$ and $\omega$ are rationally independent, then $\rho\left(s_{E}\right)$ is strictly monotone in $E$. We would like to stress that analogue results were first established for the continuous Schrödinger equation with almost periodic potentials in the fundamental paper [5].

In contrast to the situation for the Harper map and despite a large number of numerical studies (6 gives a good overview and further reference), there hardly exist any rigorous results about other parameter families of qpf circle maps. The most prominent example is probably the qpf Arnold circle map

$$
f_{\alpha, \beta, \tau}: \mathbb{T}^{2} \mapsto \mathbb{T}^{2}, \quad(\theta, x) \mapsto\left(\theta+\omega, x+\tau+\frac{\alpha}{2 \pi} \sin (2 \pi x)+\beta g(\theta) \bmod 1\right)
$$

with parameters $\alpha \in[0,1], \tau, \beta \in \mathbb{R}$ and continuous forcing function $g: \mathbb{T}^{1} \rightarrow \mathbb{R}$. This map was first studied in [7, where it was proposed as a simple model of an oscillator forced at two incommensurate frequencies. It was observed numerically that

\footnotetext{
${ }^{1}$ The presence of $p, q$-invariant graphs forces $\omega, \rho(f)$ and 1 to be rationally dependent; see Theorem 2.3 Hence, the second statement is an immediate consequence of the first.
} 
the fibred rotation number stays constant on open regions in the $(\alpha, \tau)$-parameter space, the so-called Arnold tongues (see also [8, 9, 10])2 Although this was clearly expected and backed up by numerical evidence, it was not rigorously known that the rotation numbers have to be rationally related inside these tongues and that the boundaries of the tongues are always given by continuous curves. Now these two facts are direct consequences of Theorems A and B, which more generally imply the following

Corollary 1.3. Let $\mathcal{A}$ be a topological space and suppose $\left(f_{\alpha, \tau}\right)_{(\alpha, \tau) \in \mathcal{A} \times \mathbb{R}}$ is a pa-

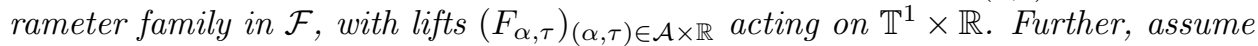
$F_{\alpha, \tau}$ depends continuously on $(\alpha, \tau)$ and strictly monotonically on $\tau$, in the sense that $F_{\alpha, \tau, \theta}(x)<F_{\alpha, \tau^{\prime}, \theta}(x) \forall(\theta, x) \in \mathbb{T}^{1} \times \mathbb{R}$ whenever $\tau<\tau^{\prime}$. For any $\rho \in \mathbb{R}$, define the boundaries of the corresponding Arnold tongue by

$\tau_{\rho}^{-}(\alpha):=\inf \left\{\tau \in \mathbb{R} \mid \rho\left(F_{\alpha, \tau}\right)=\rho\right\} \quad$ and $\quad \tau_{\rho}^{+}(\alpha):=\sup \left\{\tau \in \mathbb{R} \mid \rho\left(F_{\alpha, \tau}\right)=\rho\right\}$.

Then there holds:

(i) $\tau_{\rho}^{-}=\tau_{\rho}^{+}$whenever $\omega, \rho$ and 1 are rationally independent.

(ii) For all $\rho \in \mathbb{R}$, the functions $\tau_{\rho}^{ \pm}: \mathcal{A} \rightarrow \mathbb{R}$ are continuous.

Note that statement (i) follows directly from Theorem A. In order to obtain (ii), it suffices to remark that the three sets $\left\{(\alpha, \tau) \mid \tau<\tau_{\rho}^{-}(\alpha)\right\},\left\{(\alpha, \tau) \mid \tau>\tau_{\rho}^{+}(\alpha)\right\}$ and $\left\{(\alpha, \tau) \mid \tau_{\rho}^{-}(\alpha)<\tau<\tau_{\rho}^{+}(\alpha)\right\}$ are all open. For the first two sets, this holds simply because the rotation number $\rho(F)$ depends continuously on $F$, and for the third set this is a consequence of Theorem $\mathrm{B}$, since the situation described there is clearly topologically stable.

Finally, we want to mention that Theorem A may also yield interesting information in situations with rationally related rotation numbers: The simulations in [8, 9] suggest that for certain parameters $\alpha, \beta$ in (1.4), the $\tau$-interval with fibred rotation number zero is collapsed to a single point. This observation is confirmed in [11] by showing that for suitable forcing function $g$ and parameters $\alpha, \beta$ the map $f_{\alpha, \beta, 0}$ has fibred rotation number zero but minimal dynamics and therefore has no $p, q$-invariant strips. Consequently $\tau \mapsto \rho\left(f_{\alpha, \beta, \tau}\right)$ is strictly monotone in $\tau=0$ by Theorem A.

\section{Preliminaries}

For any set $A \subseteq \mathbb{T}^{2}$ and $\theta \in \mathbb{T}^{1}$ we let $A_{\theta}:=\left\{x \in \mathbb{T}^{1} \mid(\theta, x) \in A\right\}$. In the simplest case $p=q=1$, the definition of an invariant strip is as follows:

Definition 2.1. Let $f \in \mathcal{F} . A \subseteq \mathbb{T}^{2}$ is called a 1,1-invariant strip if it is compact, $f$-invariant and for all $\theta \in \mathbb{T}^{1}$ the set $A_{\theta}$ consists of exactly one nontrivial interval (i.e. $\neq \emptyset$ or $\mathbb{T}^{1}$ ).

Note that in particular this definition includes continuous invariant curves. As the formulation for the general case $(p, q) \in \mathbb{N}^{2}$ is slightly technical and we actually do not have to use it, we refrain from giving the precise definition here and refer to 2 ] or [3]. However, in order to get a basic idea one should think of $p p$-periodic closed curves that are permuted by the action of $f$ and that all wind around the torus $q$ times in the $\theta$-direction. The union of these curves intersects every fibre

\footnotetext{
${ }^{2}$ For the unforced Arnold circle map, this is certainly well-known.
} 
in exactly in $p q$ points, and roughly spoken one allows each of these points to be replaced by a closed interval in the definition of a $p, q$-invariant strip.

The important thing we have to know here is the fact that the existence of a $p, q$-invariant strip $A$ forces the fibred rotation number to be of the form

$$
\rho(f)=\frac{k}{q} \omega+\frac{l}{p q} \bmod 1,
$$

where the integers $k, l, p$ and $q$ are determined by the topological and dynamical structure of $A$ and vice versa (see Lemma 3.9 in [2]). Further, by going over to a suitable iterate, lifting the system to a $q$-fold cover $(\mathbb{R} / q \mathbb{Z}) \times \mathbb{T}^{1}$ and rescaling, one can always transform a system with a $p, q$-invariant graph into one with a 1,1 invariant graph (compare Lemma 2.15 in $\underline{3}$; this is the reason why we do not need the general definition). For the latter (2.1) implies that the fibred rotation number will be of the form $\rho(f)=k \omega \bmod 1$, and by conjugating with $h:(\theta, x) \mapsto(\theta, x-k \theta)$ we can finally assume that $\rho(f)=0$.

The proof of Theorem A is based on a classification result for qpf circle homeomorphisms [3]. In order to state it, we have to introduce the 'deviations from the constant rotation', which are defined as

$$
D_{F}(n, \theta, x):=F_{\theta}^{n}(x)-x-n \rho(F) .
$$

In contrast to the unforced situation, these deviations do not have to be uniformly bounded in $(n, \theta, x)$ in the forced case. However, if the deviations are unbounded, they have to be unbounded on every single orbit (see Theorem 1.3 in [10]). This motivates the following definition:

Definition 2.2. $f \in \mathcal{F}$ is called $\rho$-bounded if the deviations are uniformly bounded and $\rho$-unbounded otherwise.

Note that the boundedness of the deviations does not depend on the choice of the lift $F$. We also remark that, again by Theorem 1.3 in 10, in the $\rho$-unbounded case there always exist orbits for which the deviations are unbounded from above (and similarly from below). It turns out that in the $\rho$-bounded case, the natural analogue of the Poincaré Classification Theorem (e.g. [12]) holds:

Theorem 2.3 (Theorems 3.1 and 4.1 in [3]). If $f \in \mathcal{F}$ is $\rho$-bounded, then either there exists a p,q-invariant strip and $\rho(f), \omega$ and 1 are rationally dependent or $f$ is semi-conjugate to the irrational torus translation $(\theta, x) \mapsto(\theta+\omega, x+\rho(f))$ by a semi-conjugacy $h$ which is fibre-respecting (i.e. $\pi_{1} \circ h=\pi_{1}$ ). If $f \in \mathcal{F}$ is $\rho$ unbounded, then neither of these two alternatives can occur and the map is always topologically transitive.

Examples of systems with $\rho$-unbounded behaviour can be found e.g. in [1], similar examples with minimal dynamics in 13 .

For the proof of Theorem B we need some notions and facts concerning qpf monotone maps. We call $F: \mathbb{T}^{1} \times \mathbb{R} \rightarrow \mathbb{T}^{1} \times \mathbb{R}$ a qpf monotone map if it is continuous, has skew product structure as in (1.1) and all fibre maps $F_{\theta}: \mathbb{R} \rightarrow \mathbb{R}$ are monotonically increasing. In particular, this is true if $F$ is the lift of some $f \in \mathcal{F}$.

An $F$-invariant graph is a measurable function $\varphi: \mathbb{T}^{1} \rightarrow \mathbb{R}$ which satisfies

$$
F_{\theta}(\varphi(\theta))=\varphi(\theta+\omega) \quad \forall \theta \in \mathbb{T}^{1} .
$$


For any compact set $K \subseteq \mathbb{T}^{1} \times \mathbb{R}$ with $\pi_{1}(K)=\mathbb{T}^{1}$, the upper and lower bounding graphs are defined as

$$
\varphi_{K}^{+}(\theta):=\sup K_{\theta} \quad \text { and } \quad \varphi_{K}^{-}(\theta):=\inf K_{\theta},
$$

respectively. Due to compactness, $\varphi_{K}^{+}$will be upper semi-continuous and $\varphi_{K}^{-}$lower semi-continuous. Furthermore, if $K$ is $F$-invariant, then $\varphi_{K}^{ \pm}$are $F$-invariant graphs. A compact set $K$ is called pinched if there exists some $\theta \in \mathbb{T}^{1}$ with $\varphi_{K}^{-}(\theta)=\varphi_{K}^{+}(\theta)$. We remark that the set of $\theta$ 's with the latter property is always either empty or residual [14. The following result will be useful:

Theorem 2.4 (Theorem 2 in 14). Suppose $F$ is a qpf monotone map and $K$ is a compact set which contains only one minimal set. Then $K$ is pinched.

Finally, for any two graphs $\varphi, \psi: \mathbb{T}^{1} \rightarrow \mathbb{R}$ with $\varphi \leq \psi$ we will use the notation

$$
[\varphi, \psi]:=\{(\theta, x) \mid x \in[\varphi(\theta), \psi(\theta)]\},
$$

and similarly for open and half-open intervals.

\section{Strict monotonicity: Proof of Theorem A}

From now on, we fix $f \in \mathcal{F}$ with lift $F_{0}$ and let $F_{\varepsilon}$ be as is Definition 1.1 Theorem 2.3 allows us to divide the problem into two cases, namely the $\rho$-unbounded one and the one with a semi-conjugacy to an irrational translation. We treat them separately in the following two lemmas, starting with the $\rho$-unbounded case:

Lemma 3.1. Suppose $f$ is $\rho$-unbounded. Then the rotation number is strictly monotone in $f$.

Proof. We only have to show that for any $\varepsilon>0$ there holds $\rho\left(F_{\varepsilon}\right)>\rho\left(F_{0}\right)$, as the case where $\varepsilon<0$ is symmetric. Hence, fix any $\varepsilon>0$. Due to uniform continuity, there exists $\delta>0$ such that $\left|\theta-\theta^{\prime}\right|<\delta$ implies $F_{0, \theta}(x) \leq F_{\varepsilon, \theta^{\prime}}(x) \forall x \in \mathbb{R}$. By induction and using the monotonicity of the fibre maps, we obtain

$$
F_{0, \theta}^{n}(x) \leq F_{\varepsilon, \theta^{\prime}}^{n}(x) \quad \forall \theta, \theta^{\prime}:\left|\theta-\theta^{\prime}\right|<\delta \forall x \in \mathbb{R}, n \in \mathbb{N} .
$$

Fix $N \in \mathbb{N}$ such that $\{n \omega\}_{n=0}^{N}$ is $\delta$-dense in the circle. As we mentioned above, in the $\rho$-unbounded case there always exists an orbit $\left(\theta_{0}, x_{0}\right)$ on which the deviations are unbounded from above. Let $\theta_{k}:=\theta_{0}+k \omega$. Since all maps $F_{\theta}^{n}$ are lifts of circle homeomorphisms, there obviously holds

$$
\left|D(n, \theta, x)-D\left(n, \theta, x^{\prime}\right)\right| \leq 1 \quad \forall n \in \mathbb{N}, \theta \in \mathbb{T}^{1}, x, x^{\prime} \in \mathbb{R} .
$$

Further, there exists a constant $C>0$ which satisfies

$$
|D(n, \theta, x)-D(n+m, \theta, x)| \leq C \quad \forall n \in \mathbb{N}, \theta \in \mathbb{T}^{1}, x \in \mathbb{R} \text { and } m=1, \ldots, N .
$$

As the deviations of the orbit of $\left(\theta_{0}, x_{0}\right)$ are unbounded from above, we can choose $M \in \mathbb{N}$ with $D\left(M, \theta_{0}, x_{0}\right) \geq C+2$. Together with (3.3) this implies

$$
D\left(M, \theta_{k}, x\right) \geq 1 \quad \forall k=0, \ldots, N, x \in \mathbb{R} .
$$

Now we proceed inductively to show that for any $\theta \in \mathbb{T}^{1}, x \in \mathbb{R}$ there holds

$$
F_{\varepsilon, \theta}^{n M}(x)-x \geq n\left(M \rho\left(F_{0}\right)+1\right) \quad \forall n \geq 0,
$$


which immediately implies $\rho\left(F_{\varepsilon}\right) \geq \rho\left(F_{0}\right)+\frac{1}{M}$. For $n=0$ there is nothing to prove, so assume the statement holds for some $n \geq 0$. Let $x^{\prime}:=F_{\varepsilon, \theta}^{n M}(x)$ and choose $k \in\{0, \ldots, N\}$ such that $\left|\theta+n M \omega-\theta_{k}\right|<\delta$ (recall the choice of $\left.N\right)$. Then

$$
\begin{aligned}
F_{\varepsilon, \theta}^{(n+1) M}(x)-x & =F_{\varepsilon, \theta+n M \omega}^{M}\left(x^{\prime}\right)-x^{\prime}+x^{\prime}-x \\
& \stackrel{\text { (3.1) }}{\geq} F_{0, \theta_{k}}^{M}\left(x^{\prime}\right)-x^{\prime}+n\left(M \rho\left(F_{0}\right)+1\right) \\
& =M \rho\left(F_{0}\right)+D\left(M, \theta_{k}, x^{\prime}\right)+n\left(M \rho\left(F_{0}\right)+1\right) \\
& \stackrel{\text { (3.4) }}{\geq}(n+1)\left(M \rho\left(F_{0}\right)+1\right) .
\end{aligned}
$$

This only leaves the case where $f$ is semi-conjugate to an irrational torus translation. In order to treat this, it is convenient to look at invariant measures. Let $h:(\theta, x) \mapsto\left(\theta, h_{\theta}(x)\right)$ be the fibre-respecting semi-conjugacy from Theorem 2.3 and define a measure $\mu$ on $\mathbb{T}^{2}$ by $\mu(A)=\operatorname{Leb}^{2}(h(A)) \forall A \in \mathcal{B}\left(\mathbb{T}^{2}\right)$, where $\operatorname{Leb}^{n}$ denotes the Lebesgue measure on $\mathbb{T}^{n}$. Then it is easy to see that $\mu$ is an ergodic $f$-invariant probability measure. (In fact, it can be deduced from Theorem 4.1 in [15] that $\mu$ is the only $f$-invariant probability measure in this situation.) From the definition of $\mu$ it follows that

$$
\mu(A)=\int_{\mathbb{T}^{1}} \mu_{\theta}\left(A_{\theta}\right) d \theta \quad \forall A \in \mathcal{B}\left(\mathbb{T}^{2}\right)
$$

where $A_{\theta}:=\left\{x \in \mathbb{T}^{1} \mid(\theta, x) \in A\right\}$ and the so-called fibre measures (or conditional measures) are defined as $\mu_{\theta}(B)=\operatorname{Leb}^{1}\left(h_{\theta}(B)\right) \forall B \in \mathcal{B}\left(\mathbb{T}^{1}\right)$. Obviously, by this definition the measures $\mu_{\theta}$ are all continuous (in the sense that $\mu_{\theta}(\{x\})=0 \forall x \in$ $\mathbb{T}^{1}$ ) and $f_{\theta}$ maps $\mu_{\theta}$ to $\mu_{\theta+\omega}$, i.e. $\mu_{\theta+\omega}=f_{\theta}^{*} \mu_{\theta}$. (A general discussion of fibre measures can for example be found in [16.) Therefore the strict monotonicity of the rotation number in the semi-conjugated case is a consequence of the following lemma.

Lemma 3.2. Suppose $f$ has an ergodic invariant probability measure $\mu$ with continuous fibre measures $\mu_{\theta}$. Then the rotation number is strictly monotone in $f$. In particular, this is true whenever $f$ is semi-conjugate to an irrational translation of the torus.

Proof. Fix $\varepsilon>0$. We identify $\mu$ and $\mu_{\theta}$ with their natural lifts to $\mathbb{T}^{1} \times \mathbb{R}$ and $\mathbb{R}$, respectively. As the fibrewise rotation number does not depend on $(\theta, x)$, it suffices to show that there exists one $(\theta, x) \in \mathbb{T}^{1} \times \mathbb{R}$ such that

$$
\lim _{n \rightarrow \infty} \frac{1}{n} F_{\varepsilon, \theta}^{n}(x)>\lim _{n \rightarrow \infty} \frac{1}{n} F_{0, \theta}^{n}(x) .
$$

Let us first see that (3.7) is a consequence of the following statement:

$$
p(\theta):=\min \left\{p \in \mathbb{N} \mid F_{\varepsilon, \theta}^{p}(x) \geq F_{0, \theta}^{p}(x)+1 \forall x \in \mathbb{R}\right\}<\infty \text { for Leb }{ }^{1} \text {-a.e. } \theta \in \mathbb{T}^{1} .
$$

Indeed, (3.8) implies that for some $q \in \mathbb{N}$ the set $A_{q}:=\left\{\theta \in \mathbb{T}^{1} \mid p(\theta)=q\right\}$ has positive measure. Without loss of generality we can assume that $q=1$, otherwise we replace $f$ by its $q$ th iterate. Due to the monotonicity and periodicity of the fibre maps we obtain

$$
F_{\varepsilon, \theta}^{n}(x) \geq F_{0, \theta}^{n}(x)+\sum_{i=0}^{n-1} \mathbf{1}_{A_{1}}(\theta+i \omega)
$$


As $\theta \mapsto \theta+\omega$ is ergodic, this implies

$$
\lim _{n \rightarrow \infty} \frac{1}{n} F_{\varepsilon, \theta}(x) \geq \lim _{n \rightarrow \infty} \frac{1}{n} F_{0, \theta}^{n}(x)+\operatorname{Leb}^{1}\left(A_{1}\right)>\lim _{n \rightarrow \infty} \frac{1}{n} F_{0, \theta}^{n}(x)
$$

for Leb $^{1}$-a.e. $\theta$, thus proving (3.7).

It remains to show that the function $p$ is $\operatorname{Leb}^{1}$-a.s. finite. To that end, note that for any $\varepsilon>0$ the function

$$
g(\theta, x):=\mu_{\theta}([x, x+\varepsilon))
$$

is $\mu$-a.s. strictly positive. Therefore

$$
\delta:=\frac{1}{2} \int_{\mathbb{T}^{2}} g d \mu>0 .
$$

By Birkhoff's Ergodic Theorem, for $\mu$-a.e. $(\theta, x)$ we have

$$
\lim _{n \rightarrow \infty} \frac{1}{n} \sum_{i=0}^{n-1} g \circ f^{i}(\theta, x)=2 \delta,
$$

and from (3.6) it follows that (3.9) holds $\mu_{\theta^{-}}$a.s. for Leb $^{1}$-a.e. fixed $\theta$. Let $S \subseteq \mathbb{T}^{1}$ be a set of measure $\operatorname{Leb}^{1}(S)=1$ which is invariant under rotation by $\omega$ and has the property that (3.9) holds for $\mu_{\theta}$-a.e. $x \in \mathbb{T}^{1}$ whenever $\theta \in S$. Then for every $\theta \in S$ and $\mu_{\theta}$-a.e. $x$ there holds

$$
\kappa(\theta, x):=\min \left\{k \in \mathbb{N} \mid g \circ f^{k}(\theta, x)>\delta\right\}<\infty .
$$

We will show that $p$ only takes finite values on $S$. In order to do so, we fix $\theta_{0} \in S$ and $x_{0} \in \mathbb{R}$ such that $\left.\kappa\left(\theta_{0}, x_{0}\right)<\infty\right\}$ We will construct sequences $k_{i}$ and $y_{i}\left(i \in \mathbb{N}_{0}\right)$ with the following properties: If $K_{i}:=\sum_{j=0}^{i} k_{j}, \theta_{i}:=\theta_{0}+K_{i} \omega, x_{i}:=F_{0, \theta_{0}}^{K_{i}}\left(x_{0}\right)$ and $z_{i}:=F_{\varepsilon, \theta_{0}}^{K_{i}}\left(x_{0}\right)$, then

$$
\begin{aligned}
x_{i} \leq y_{i} & \leq z_{i}, \\
\mu_{\theta_{i}}\left(\left[x_{i}, y_{i}\right)\right) & \geq i \cdot \delta, \\
\kappa\left(\theta_{i}, y_{i}\right) & <\infty .
\end{aligned}
$$

In order to construct these sequences, we let $y_{0}=x_{0}$ and $k_{0}=0$. Then for $i=0$, there is nothing to show. Hence, suppose $k_{0}, \ldots, k_{i}$ and $y_{0}, \ldots, y_{i}$ with the required properties have been chosen. Let $k_{i+1}:=\kappa\left(\theta_{i}, y_{i}\right)$. Then

$$
\begin{aligned}
& \mu_{\theta_{i+1}}\left(\left[x_{i+1}, z_{i+1}\right)\right) \geq \mu_{\theta_{i+1}}\left(\left[x_{i+1}, F_{\varepsilon, \theta_{i}}^{k_{i+1}}\left(y_{i}\right)\right)\right) \\
& \quad=\mu_{\theta_{i}}\left(\left[x_{i}, y_{i}\right)\right)+\mu_{\theta_{i+1}}\left(\left[F_{0, \theta_{i}}^{k_{i+1}}\left(y_{i}\right), F_{\varepsilon, \theta_{i}}^{k_{i+1}}\left(y_{i}\right)\right)\right)>i \delta+\delta .
\end{aligned}
$$

Thus we can choose some $y_{i+1} \in\left(x_{i+1}, z_{i+1}\right)$ which satisfies (3.11) and (3.12).

If $i>1 / \delta$, then (3.11) gives

$$
\mu_{\theta_{i}}\left(\left[x_{i}, z_{i}\right)\right) \geq \mu_{\theta_{i}}\left(\left[x_{i}, y_{i}\right)\right)>1
$$

and consequently, as $\mu_{\theta}([x, x+1))=1 \forall(\theta, x)$ (recall that $\mu$ is a probability measure),

$$
F_{\varepsilon, \theta_{0}}^{K_{i}}\left(x_{0}\right)>F_{0, \theta_{0}}^{K_{i}}\left(x_{0}\right)+1 .
$$

This in turn implies $p\left(\theta_{0}\right) \leq K_{i}$. As $\theta_{0} \in S$ was arbitrary and $\operatorname{Leb}^{1}(S)=1$, this shows (3.8), and thus completes the proof.

\footnotetext{
${ }^{3}$ Here we also apply $g$ and $\kappa$ to points $(\theta, x) \in \mathbb{T}^{1} \times \mathbb{R}$ by identifying $x \in \mathbb{R}$ with its projection to $\mathbb{T}^{1}$.
} 


\section{Mode-locking: Proof of Theorem B}

For $f \in \mathcal{F}$ with lift $F_{0}$, let $F_{\varepsilon}$ be as is Definition[1.1. Due to Theorem A, we only have to consider the case where $f$ has a $p, q$-invariant strip. Further, as mentioned in Section 2 we can always assume that $p=q=1$ and $\rho(f)=0$. In this situation, the statement of Theorem B is a consequence of the following:

Proposition 4.1. Suppose $\rho(f)=\rho\left(F_{0}\right)=0$ and for some $\varepsilon>0$ there holds $\rho\left(F_{\varepsilon}\right)=0$. Then there exists a continuous curve $\gamma^{+}: \mathbb{T}^{1} \rightarrow \mathbb{R}$, which is mapped strictly below itself by some iterate of $F_{0}$. Similarly, if $\rho\left(F_{-\varepsilon}\right)=0$, then there exists a curve $\gamma^{-}$which is mapped strictly above itself by some iterate of $F_{0}$.

For if $f$ is mode-locked, then we obtain two curves $\gamma^{+}$and $\gamma^{-}$which are mapped strictly above, respectively below, themselves by some iterate of $F_{0}$. It is easy to see that $\gamma^{+}$and $\gamma^{-}$must be disjoint and that they are lifts of the continuous boundaries of a closed annulus $\mathcal{A} \subseteq \mathbb{T}^{2}$ which is mapped inside its own interior by some iterate of $f$.

Proof of Proposition 4.1. Fix $f \in \mathcal{F}$ with lift $F_{0}$ and suppose $\rho\left(F_{0}\right)=0$ and $\rho\left(F_{\varepsilon}\right)=0$ for some $\varepsilon>0$. We note that in this case the map $F_{\varepsilon}$ must have bounded orbits. Otherwise the mapping $\varepsilon^{\prime} \mapsto \rho\left(F_{\varepsilon^{\prime}}\right)$ would be strictly monotone in $\varepsilon$, due to Lemma 3.1, contradicting $\rho\left(F_{0}\right)=\rho\left(F_{\varepsilon}\right)$. (Note that $F_{\varepsilon}$ is the lift of a qpf circle homeomorphism as well.)

Since orbits are bounded, it follows that there exist compact $F_{\varepsilon}$-invariant sets. Suppose that $K$ is compact, $F_{\varepsilon}$-invariant and in addition minimal. Then $K$ is pinched by Theorem 2.4 and consequently there exist $\theta_{0} \in \mathbb{T}^{1}$ and $\delta>0$ such that $\varphi_{K}^{+}(\theta)<\varphi_{K}^{-}(\theta)+\varepsilon \forall \theta \in B_{\delta}\left(\theta_{0}\right)$. This implies that for any $(\theta, x) \in K$ there exists an integer $n=n(\theta, x)>0$ such that the point $F_{0}^{n}(\theta, x)$ lies strictly below the set $K$ in the sense that $F_{0, \theta}^{n}(x)<\varphi_{K}^{-}(\theta+n \omega)$. (In fact, we can choose $n(\theta, x)$ as the smallest integer $n \geq 1$ which satisfies $\theta+(n-1) \omega \in B_{\delta}\left(\theta_{0}\right)$.)

As the set $\left[-\infty, \varphi_{K}^{-}\right)$is open and due to compactness of $K$, this further yields that there exists some integer $N>0$ such that $F_{0}^{N}$ maps the set $K$ strictly below itself, in the sense that

$$
\varphi_{F_{0}^{N}(K)}^{+}(\theta)<\varphi_{K}^{-}(\theta) \quad \forall \theta \in \mathbb{T}^{1}
$$

Now, the set $U:=\left\{(\theta, x) \in \mathbb{T}^{1} \times \mathbb{R} \mid \varphi_{F_{0}^{N}(K)}^{+}(\theta)<x<\varphi_{K}^{-}(\theta)\right\}$ projects down to all of $\mathbb{T}^{1}$ and is open due to the semi-continuity of the bounding graphs. Using compactness of $\mathbb{T}^{1}$, we can therefore find $\theta_{0}<\theta_{1}<\ldots<\theta_{n-1}<\theta_{n}=\theta_{0} \in \mathbb{T}^{1}$ and $y_{1}, \ldots, y_{n} \in \mathbb{R}$ such that the horizontal segments $W_{i}=\left[\theta_{i-1}, \theta_{i}\right] \times\left\{y_{i}\right\}$ are contained in $U$ for all $i=1, \ldots, n$. As $U_{\theta}$ is an open interval for all $\theta \in \mathbb{T}^{1}$, the vertical segments $V_{i}$ joining $\left(\theta_{i}, y_{i}\right)$ and $\left(\theta_{i}, y_{i+1}\right)$ are equally contained in $U$, and consequently the same is true for the union $\tilde{\Gamma}:=\bigcup_{i=1}^{n} W_{i} \cup V_{i}$. It is easy to see that $\tilde{\Gamma}$ is the graph of an essential simple closed curve in the annulus. Due to the vertical segments, $\tilde{\Gamma}$ itself cannot be represented as the graph of a continuous function $\mathbb{T}^{1} \rightarrow \mathbb{R}$. However, as $U$ is open, we may slightly tilt these vertical segments in order to obtain a continuous curve $\gamma^{+}: \mathbb{T}^{1} \rightarrow \mathbb{R}$ whose graph is still contained in $U$. Since $U$ is mapped strictly below itself due to (4.1), the same is true for $\gamma^{+}$.

Remark 4.2.

(1) As we reduced the problem to the situation with fibred rotation number 0 , the closed annulus from Proposition 4.1 will be of 'homotopy type' $(1,0)$, meaning 
that it only winds once around the torus, in the $\theta$-direction. In the general case, by redoing the transformations described in Section 2, we obtain a closed annulus of 'homotopy type' $(q, k)$ with $q$ and $k$ as in (2.1).

(2) Proposition 4.1 also contains the description of the one-sided cases: Suppose the rotation number only remains constant on one side; for example $\rho\left(F_{\varepsilon}\right)=0$ for some $\varepsilon>0$ but $\rho\left(F_{\varepsilon^{\prime}}\right)<0 \forall \varepsilon^{\prime}<0$. Then the proposition still yields the existence of a closed curve $\gamma^{+}$which is mapped below itself by some iterate of $F_{0}$. The iterates of $\gamma^{+}$form a monotonically decreasing sequence of continuous curves. In the limit, they have to converge to an upper semi-continuous graph $\varphi^{+}$, which is the upper boundary of an invariant strip. Projecting the situation to the torus, we obtain the existence of a closed curve which is mapped towards some 1,1-invariant strip in the clockwise direction. (Note that 'being mapped above or below itself' does not make any sense on the torus, unless there is some kind of reference object.) As there is no curve which is mapped towards this strip in the counterclockwise direction, the situation resembles the one with a parabolic periodic orbit in the one-dimensional case. Again, this picture remains valid in the case of a general $p, q$-invariant strip; only the homotopy type of all objects involved changes.

\section{ACKNOWLEDGEMENTS}

This cooperation started during a workshop on 'Dynamics of cocycles and onedimensional spectral theory' at Oberwohlfach 2005. We would like to thank the organisers David Damanik, Russell Johnson and Daniel Lenz for making this possible. The first author was partially supported by SVeFUM. The second author was supported by grant Ke 514/6-1 of the German Research Foundation (DFG), which also funded a visit of the first author to Erlangen.

\section{REFERENCES}

[1] M. Herman. Une méthode pour minorer les exposants de Lyapunov et quelques exemples montrant le caractère local d'un théorème d'Arnold et de Moser sur le tore de dimension 2. Commentarii Mathematici Helvetici, 58:453-502, 1983. MR727713 (85g:58057)

[2] T. Jäger and G. Keller. The Denjoy type-of argument for quasiperiodically forced circle diffeomorphisms. Ergodic Theory and Dynamical Systems, 26(2):447-465, 2006. MR2218770 (2007b:37081)

[3] T. Jäger and J. Stark. Towards a classification of quasiperiodically forced circle homeomorphisms. Journal of the LMS, 73(3):727-744, 2005. MR2241977 (2008f:37087)

[4] F. Delyon and B. Souillard. The rotation number for finite difference operators and its properties. Commununications in Mathematical Physics, 89:415-426, 1983. MR709475 (85d:60123)

[5] R. Johnson and J. Moser. The rotation number for almost periodic potentials. Communications in Mathematical Physics, 84:403-438, 1982. MR667409 (83h:34018)

[6] A. Prasad, S. Negi, and R. Ramaswamy. Strange nonchaotic attractors. International Journal of Bifurcation and Chaos, 11(2):291-309, 2001. MR:1830343 (2002b:37044)

[7] M. Ding, C. Grebogi and E. Ott. Evolution of attractors in quasiperiodically forced systems: From quasiperiodic to strange nonchaotic to chaotic. Physical Review A, 39(5):2593-2598, 1989.

[8] U. Feudel, J. Kurths and A. Pikovsky. Strange nonchaotic attractor in a quasiperiodically forced circle map. Physica D, 88:176-186, 1995. MR1360883 (96j:58146)

[9] P. Glendinning, U. Feudel, A.S. Pikovsky, and J. Stark. The structure of mode-locked regions in quasi-periodically forced circle maps. Physica D, 140:227-243, 2000. MR:1757089 (2001b:37060)

[10] J. Stark, U. Feudel, P. Glendinning, and A. Pikovsky. Rotation numbers for quasiperiodically forced monotone circle maps. Dynamical Systems, 17(1):1-28, 2002. MR 1888695 (2003h:37058) 
[11] T. Jäger. Strange non-chaotic attractors in quasiperiodically forced circle maps. Preprint 2007.

[12] A. Katok and B. Hasselblatt. Introduction to the Modern Theory of Dynamical Systems. Cambridge University Press, 1997. MR.1326374 (96c:58055)

[13] K. Bjerklöv. Positive Lyapunov exponent and minimality for a class of one-dimensional quasiperiodic Schrödinger equations. Ergodic Theory and Dynamical Systems, 25:1015-1045, 2005. MR2158395 (2007c:47035)

[14] J. Stark. Transitive sets for quasiperiodically forced monotone maps. Dynamical Systems, 18(4):351-364, 2003. MR2021504 (2004k:37086)

[15] H. Furstenberg. Strict ergodicity and transformation of the torus. American Journal of Mathematics, 83:573-601, 1961. MR0133429 (24:A3263)

[16] L. Arnold. Random Dynamical Systems. Springer, 1998. MR.1723992 (2000m:37087)

[17] R. Johnson. On a Floquet theory for almost-periodic, two-dimensional linear systems. $J$. Differential Equations, 37(2):184-205, 1980. MR587221 (81j:58069)

Department of Mathematics, University of Toronto, Toronto, Ontario, Canada M5S 3G4

E-mail address: bjerklov@math.utoronto.ca

Department of Mathematics, Collège de France, 3 Rue d'Ulm, 75005 Paris, France

E-mail address: tobias.jager@college-de-france.fr 